\title{
Introducción de la topología de vecindades en los trabajos de Fréchet y Hausdorff
}

\author{
Luis Carlos Arboleda \\ Universidad del Valle, Cali
}

Artículo de posesión para el ingreso como miembro de número de la

Academia Colombiana de Ciencias Exactas, Físicas y Naturales el 26 de abril de 2017

\section{Resumen}

En la primera parte, retomamos testimonios de Fréchet sobre la naturaleza de sus primeros trabajos (1904-1906) en los campos emergentes del Análisis funcional y Análisis general, en relación con su idea de introducir una estructura topológica en un espacio abstracto. En la segunda parte, destacamos la influencia que tuvo en esta idea, el punto de vista algebraico de la época de extender las nociones cantorianas a un espacio abstracto con una estructura de grupo finito. Fréchet supo aprovechar técnicas como el "modo de composición" entre los elementos del espacio, para axiomatizar operaciones y estructuras de la "clase $L$ " con convergencia secuencial, la "clase $V$ " con sistema de vecindades, la "clase E" con "écart" (métrica). Luego se aprovechan nuevos datos históricos para reafirmar la proximidad de las concepciones filosóficas subyacentes a estas investigaciones, con las ideas de Leibniz, específicamente en cuanto al método de "análisis de los principios". En la tercera parte se estudia la contribución de Hausdorff de 1912 y 1914 al establecimiento de la axiomática de las vecindades para la topología de un espacio abstracto. Teniendo en cuenta las observaciones de Weyl y Bourbaki de que Hausdorff se inspiró para ello en Hilbert, se examina el sistema de axiomas para las vecindades del plano introducido por Hilbert en dos trabajos de 1902 consagrados al problema de la continuidad del espacio. Se exploran las conexiones del "espacio topológico" de Hausdorff basado en las vecindades, con las nociones de métrica, convergencia secuencial y vecindades propuestas años antes por Fréchet. Hausdorff insistió desde el comienzo que la topología del espacio separable tenía las características de generalidad y rigor formal que le permitían adaptarse a las aplicaciones mejor que otras. Se mostrará que todo ello era consistente con los ideales de simplicidad, unidad y economía de pensamiento que Hausdorff había adquirido en sus trabajos filosóficos tempranos. (C) 2017. Acad. Colomb. Cienc. Ex. Fis. Nat.

Palabras Clave: Historia de la topología; Filosofía de la práctica matemática; Sistemas de axiomas para vecindades; Fréchet; Hausdorff.

\section{Introduction of the topology structure in Fréchet and Hausdorff works}

\begin{abstract}
In the first part, we remind Fréchet's testimonies about the approach of his early work (1904-1906) in the emerging fields of Functional Analysis and General Analysis, in relation to his idea of introducing a topological structure in an abstract space. In the second part, we highlight the influence that had on this idea, the algebraic point of view of the time of extending the Cantorian notions to an abstract space with a finite group structure. Fréchet took advantage of techniques such as the "composition mode" between the elements of the space, to axiomatize operations and structures of the "class" with sequential convergence, the "class" with neighborhood system, the "class" with "écart" (metric). Then, new historical data are used to reaffirm the proximity of the philosophical conceptions underlying these investigations with the ideas of Leibniz, specifically with regard to the method of "analysis of principles". The third part examines the contribution of Hausdorff of 1912 and 1914 to the establishment of the neighborhood axiomatics for the topology of an abstract space. Taking into account the observations of Weyl and Bourbaki that Hausdorff drew on Hilbert, we examine the system of axioms for the neighborhoods of the plane introduced by Hilbert in two 1902 papers devoted to the problem of the space continuity. As regards Fréchet's influence in Hausdorff, we explore the connections of Hausdorff's "topological space" based on neighborhoods with the notions of metric, sequential convergence and neighborhoods, proposed years earlier by Fréchet. From the beginning, Hausdorff argued that the topology of the separable space had the characteristics of generality and formal rigor that allowed it to adapt to applications better than others. It is shown that all this was consistent with the ideals of simplicity, unity and economy of thought Hausdorff had acquired in his early philosophical works. (C) 2017. Acad. Colomb. Cienc. Ex. Fis. Nat.
\end{abstract}

Key Words: History of topology; Philosophy of mathematical practice; systems of axioms for neighborhoods; Fréchet; Hausdorff.

Correspondencia:

Luis Carlos Arboleda, luis.carlos.arboleda@gmail.com; Recibido: 19 de julio de 2017; Aceptado: 15 de diciembre de 2017 


\section{Introducción}

Es bien sabido que los conceptos y métodos fundamentales de la topología conjuntista relativos a la recta real y a los espacios euclidianos se encuentran en la creación cantoriana de finales del siglo XIX. Con posterioridad a los trabajos de Cantor otros matemáticos se consagraron a generalizar estos resultados a espacios de naturaleza cualquiera, dando lugar a la constitución de nuevos campos matemáticos. En particular, la Topología conjuntista o Point-Set Topology se fue configurando rápidamente y hacia los años 1920 las investigaciones en este campo registraban un gran avance, sobre todo en conexión con aquellos dominios que posibilitaron su existencia, el Análisis y el Análisis funcional (Arboleda, 1980a) (Arboleda, 1980b) (Tarrés Freixenet, 1994).

En este trabajo examinamos algunas de las características históricas y filosóficas de este desarrollo, las cuales fueron previamente esbozadas en (Arboleda, 2012) y que aquí tratamos a fondo aprovechando una bibliografía más amplia, actualizada y original. Nos interesan en particular los ideales de la práctica matemática que más intervinieron en estas transformaciones. No entramos en el estudio de la tendencia que por la misma época se manifiesta en la síntesis de ideas y métodos conjuntistas y algebraicos, y que permitió la emergencia de la topología algebraica cuyos orígenes remontan a los trabajos geométricos de Euler y Riemann basados en métodos combinatorios (Hirsch, 1978) (Alexandroff y Fedorchuk, 1978).

En varios de sus trabajos, concretamente en (Fréchet, 1928) y (Fréchet, 1941), Fréchet recuerda que en los comienzos del Análisis funcional (sobre todo en los trabajos de Volterra y la escuela italiana), lo usual era estudiar por separado distintas categorías de funcionales numéricas según los campos particulares de definición de la variable (puntos, curvas, funciones, etc.). Una "funcional", según la denominación de Hadamard, es una función del tipo $Y=$ $F(X)$ que hace corresponder un número $Y$ a un elemento $X$ de naturaleza cualquiera.

El aporte de Fréchet consistió en reconocer que tampoco era necesario fijar la naturaleza del elemento transformado. En distintos campos de la matemática era frecuente el uso de transformaciones con valores no numéricos: transformaciones geométricas de puntos en rectas, de rectas en esferas; transformaciones en análisis de funciones en funciones, etc. En consecuencia, Fréchet se propuso adelantar el estudio de transformaciones $V=T(U)$ de elementos $U$ de naturaleza cualquiera en elementos $V$ también de naturaleza cualquiera.

Esta fue la idea de base para la constitución del nuevo campo llamado "Análisis general". En comparación con el Análisis funcional, el Análisis general no solo apuntaba a generalizar propiedades importantes del Análisis clásico. Ante todo, se proponía "llevar la abstracción hasta el límite", y unificar en un mismo campo el estudio de las propiedades comunes a la clase de funciones $V=T(U)$. En ello, Fréchet reconoce que se inspiró en los métodos generales empleados en el Cálculo vectorial y la Teoría de grupos abstractos. (Fréchet, 1941). Pero, esta tarea de generalizar objetos y propiedades del análisis clásico para darles una presentación simple y unificada, se enfrentaba a la necesidad previa de caracterizar apropiadamente en los espacios abstractos nociones fundamentales como continuidad, diferencial, integral, etc. Como lo reconocerá años después (Fréchet, 1941; 71):

"para formular propiedades que se expresan simultáneamente en términos de la variable $U$ y su transformada $V=T(U)$, es necesario determinar el sentido de ciertas nociones fundamentales relativas, de una parte, a la variable $U$ y el conjunto $E$ de sus 'valores' y, de otra parte, a la transformada $V$ y el conjunto $F$ de sus 'valores"'.

La noción de proximidad se revela, entonces, esencial para caracterizar la geometría intrínseca de los conjuntos abstractos $E$ y $F$. Es decir, establecer en ellos una estructura de espacio topológico, sea a través de las nociones de distancia entre dos puntos, la de convergencia de una sucesión de puntos o la de vecindad de un punto. Fréchet advierte que esto era tanto más apremiante en el Análisis general, que en el caso "más simple" del Análisis funcional. En efecto, siendo $U$ una función clásica y $V$ un número, la tarea se reducía a dotar de una topología al espacio abstracto $E$ o "campo funcional". La caracterización de la "topología de los campos funcionales" fue uno de los problemas más delicados en el desarrollo del Análisis funcional a comienzos del siglo XX. En (Fréchet, 1934) aparece una cita en la cual Hadamard ubica la importancia de esta problemática alrededor de 1910, y reconoce los aportes que el "joven geómetra" Fréchet venía haciendo en su estudio (Hadamard, 1912; 17):

"El continuo funcional -es decir, la multiplicidad obtenida al hacer variar continuamente una función de todas las maneras posibles, no ofrece a nuestro entendimiento, ninguna imagen simple. La intuición geométrica no nos enseña nada, a priori, al respecto. Estamos obligados a poner remedio a esta ignorancia, y solo podemos hacerlo analíticamente, creando un capítulo de la teoría de conjuntos para el uso del continuo funcional."

\section{Fréchet y la génesis de las primeras estructuras topológicas}

En su historia de los espacios completos Dugac reconoce que (Dugac, 1984; p. 18):

"Fréchet abrirá con su tesis una nueva época en el análisis, caracterizada por un florecimiento de espacios nuevos y por la búsqueda de las propiedades características de estos espacios que condujo a "estructuras" a la vez flexibles y ricas para responder a las aplicaciones más importantes en el análisis."

En efecto, Fréchet introduce en su Tesis el enfoque que permitirá dotar a un espacio abstracto de una estructura topológica. Sea una clase $L$ de elementos de naturaleza cualquiera. Fréchet define la convergencia de una sucesión $\left(a_{n}\right)$ de tales elementos con base en las siguientes condiciones (Fréchet, 1906; p. 5-6):

(i) Si $a_{n}=a$, para $n=1,2, \ldots$, entonces $\left(a_{n}\right)$ converge a $a$;

(ii) $\mathrm{Si}\left(a_{n}\right)$ converge a $a$, entonces $\left(a_{k n}\right)$ converge a $a$ para toda sucesión $\left(a_{k n}\right)$ de $\left(a_{n}\right)$. 
Es el llamado espacio $L$ de Fréchet, una clase $L$ de elementos indeterminados en la cual la convergencia de sucesiones generalizadas está determinada por las condiciones (i) y (ii).

Es interesante recordar la explicación del propio Fréchet sobre el ideal algebraico que motivó su escogencia de esta estructura (Fréchet, 1906; p. 4-5), (Taylor, 1982; p. 252). Al extender la noción de límite de sucesiones definidas en conjuntos específicos a un conjunto de elementos de naturaleza cualquiera, dice haber procedido de acuerdo con los desarrollos recientes de la teoría abstracta de grupos. Antes, las investigaciones en este campo se venían desarrollando por separado en teorías de grupos de movimientos, de sustituciones, de transformaciones, etc., en las cuales la definición del modo de composición variaba de una teoría a la otra. Pero la experimentación con distintos sistemas de axiomas en grupos específicos poco a poco condujo a la convicción de que (Fréchet, 1906; p. 5):

"Sólo era posible llegar a una teoría común absteniéndose de dar una definición general del modo de composición, y buscando las condiciones comunes a las definiciones particulares y considerando únicamente las que eran independientes de la naturaleza de los elementos considerados."

La misma idea se encuentra expuesta en la Noticia sobre sus trabajos científicos que elaboró para su ingreso a la Académie des Sciences de París. Fréchet insiste allí que el enfoque estructural empleado en sus trabajos de Topología general (Fréchet, 1933):

"evita la repetición fastidiosa de teorías y demostraciones exactamente idénticas en el fondo, aunque relativas a dominios de objetos de naturaleza diversa: números, curvas, superficies, funciones, series, grupos, variables aleatorias, etc., etc."

En el comentario de su Tesis sobre la noción de límite generalizado, Fréchet menciona de paso una referencia bibliográfica: (Séguier, 1904). Hoy sabemos que la importancia histórica de esta obra radica en la presentación de una de las primeras formulaciones sobre el carácter abstracto del concepto de grupo finito, todavía no en el marco del álgebra moderna sino de la teoría de ecuaciones polinómicas y la teoría de números. En el inicio del prefacio a sus Elementos, Séguier afirma lo siguiente:

"A partir de los diversos grupos particulares que se han encontrado en Álgebra, Análisis y Geometría, debía necesariamente emerger la idea de grupo abstracto, es decir el grupo considerado en sí mismo, independientemente de la naturaleza de sus elementos. Muchas investigaciones ya realizadas en dominios diversos se fundieron en una teoría más general que desde entonces no ha cesado de desarrollarse. Bajo el título de Elementos se encontrará aquí la parte de esta teoría que no exige ninguna representación concreta."

Séguier comienza por extender a un conjunto abstracto las nociones cantorianas requeridas por el desarrollo de la obra. Igual con respecto a las relaciones definidas entre los elementos de tales conjuntos, para las cuales se hace abstracción de su naturaleza y solo se consideran los principios lógicos comunes que reglan su comportamiento en diversos conjuntos particulares. Fréchet reconoce que el grupo abstracto se define entonces por determinado sistema de axiomas a partir de un conjunto $G$ dotado de una operación binaria $G \times G \rightarrow G$ que satisface tales axiomas. (Katz and Parshall, 2014; pp. 333-334).

El inicio de sus trabajos tendientes a extender nociones fundamentales del análisis de variable real a un conjunto abstracto, se encuentra en la nota de 1904 en la cual Fréchet define por primera vez las partes compactas $E$ del espacio $L$ como generalizaciones de los intervalos de $R$. Fréchet comienza por dotarse de una clase $C$ de elementos cualesquiera (números, superficies, etc.) (Fréchet, 1904; 849):

"en la cual es posible distinguir sus diferentes elementos. Podemos decir que $U_{A}$ es una función uniforme (u operación funcional) en un conjunto de $E$ elementos de $C$, si a todo elemento $A$ de $E$ le corresponde un número bien determinado $U_{A}$."

A partir de la noción de convergencia de sucesiones Fréchet define las de conjunto cerrado, continuidad y conjunto compacto. (Arboleda, 1984). Aunque ellas serán reelaboradas en la Tesis, le bastan en ese momento para enunciar el teorema de Weierstrass a nivel general (Fréchet, 1904; p. 849):

"TEOREMA. Toda operación funcional $U_{A}$ uniforme y continua en un conjunto compacto y cerrado $E: 1^{\circ}$ es acotada en $E ; 2^{\circ}$ obtiene al menos una vez su limite superior."

El esfuerzo de Fréchet, en este y otros trabajos, por abstraer la noción de compacto que se encontraba en estado práctico en varios contextos "naturales" de la época (Arboleda, 1984), es tanto más significativo tratándose de la generalización de un teorema del análisis como el de valores extremos, cuya importancia para el desarrollo de nuevos campos de las matemáticas había sido ya subrayada por Hilbert en su informe en memoria de Weierstrass (Hilbert, 1897; p. 333): “Con este teorema (...) Weierstrass creó un instrumento que hoy es indispensable para todos los matemáticos en las investigaciones analíticas o aritméticas más elaboradas."

En las consideraciones de método que hace al comienzo de la Tesis, Fréchet afirma que el problema de estudiar las propiedades infinitesimales de las funcionales está lógicamente precedido por el problema de establecer las propiedades infinitesimales de ciertos conjuntos abstractos. Recordemos que, en el caso más simple, se trata del problema de establecer la topología en los campos funcionales que Hadamard considera crucial en la cita al final de la Introducción de este artículo, y al cual se refiere en términos de "crear una teoría de conjuntos para el uso del continuo funcional”. (Hadamard, 1912). En el caso de los objetos del Análisis general (las transformaciones $V=$ $T(U)$ entre conjuntos $U$ y $V$ de naturaleza cualquiera), la topología se establece sobre el espacio abstracto. De ahí el título de su obra más conocida: Los espacios abstractos y su teoría considerada como introducción al Análisis general (Fréchet, 1928).

Sin embargo, Fréchet reconoce en el capítulo 2 de la Tesis que las clases $L$ son excesivamente generales y no permiten la extensión de ciertas propiedades de los conjuntos 
lineales muy en boga en su época, como la propiedad de que el conjunto derivado $E^{\prime}$, que contiene los puntos límites de un conjunto cualquiera $E$ de la clase $L$, sea cerrado. Para remediar esta situación considera necesario imponer ciertas restricciones a las clases $L$ (Fréchet, 1906; p. 17):

" $1{ }^{\circ}$ Tales restricciones deberían poder enunciarse independientemente de la naturaleza de los elementos considerados; $2^{\circ}$ Deberían satisfacerse para las clases de elementos que intervienen lo más frecuentemente en las aplicaciones; $3^{\circ}$ Deberían proporcionar la generalización que se está buscando de los teoremas sobre conjuntos lineales y funciones continuas."

Entonces define la clase $V$ ("V" de vecindad) como una clase de elementos de naturaleza cualquiera dotada de una operación binaria o función distancia que a cada par de elementos $A$ y $B$ de la clase le asigna un número llamado "vecindad" $(A, B)=(B, A) \geq 0$. La clase $V$ es una especie de clase $L$ para la cual toda sucesión $\left\{A_{n}\right\}$ de elementos de $V$ tiene como límite el elemento $A$ a condición de que $\left(A_{n}, A\right)$ $\rightarrow 0$ cuando $n \rightarrow \infty$. Esta definición garantiza que el límite es único, e igualmente tiene como consecuencia que los conjuntos derivados son cerrados (Taylor, 1982; p. 254). La estructura de la clase $V$ cumple las siguientes propiedades (Fréchet, 1906; p. 18):

(i) $(A, B)=0$ si y solamente si $A=B$;

(ii) existe una función positiva $f(\varepsilon)$, tal que $f(\varepsilon)$ tiende a 0 para $\varepsilon$ muy pequeño y para la cual $(A, C) \leq f(\varepsilon) \operatorname{si}(A, B) \leq f$ $(\varepsilon)$ y $(B, C) \leq f(\varepsilon)$ para todo $A, B, C$ de la clase $V$.

Conviene aclarar que en los años siguientes Fréchet continuó desarrollando sus investigaciones sobre la topología de las vecindades de manera independiente de Hausdorff cuya obra de 1914 solamente conoció en 1919. Estos trabajos de Fréchet, en conexión con Riesz, Moore, Hausdorff y otros autores han sido estudiados en (Arboleda, 1980). Nos interesa aquí destacar las nociones de clase $V$ separable y clase $V$ completa, las cuales van a permitirle a Fréchet desde muy temprano (1906), introducir en estos espacios de vecindades la técnica de recubrimientos de un conjunto por una familia de conjuntos.

Una clase $V$ es separable si contiene un conjunto numerable cuyo conjunto derivado es la clase. En una publicación de 1921 Fréchet modificará esta definición, y el requerimiento para la separabilidad en la clase será únicamente que existan a lo sumo conjuntos infinitos numerables que constituyan, junto con sus respectivos derivados, la clase en su totalidad. (Taylor, 1982; 255).

Luego se define la propiedad de completez de una clase $V$ sin apelar todavía a la palabra con la que habitualmente designamos el concepto (Fréchet, 1906; p. 23): "Diremos que $V$ admite una generalización del teorema de Cauchy si toda sucesión de elementos de esta clase, que satisface la condición de Cauchy, tiene un elemento límite en $V$." Y una sucesión $\left\{A_{n}\right\}$ de la clase $V$ satisface la condición de Cauchy, cuando a cada $\varepsilon>0$ corresponde un $n$ tal que $\left(A_{n}, A_{n+p}\right)<\varepsilon$, para $p=1,2,3, \ldots$

Fréchet estudia varias propiedades topológicas de las clases $V$ que darán lugar a numerosas investigaciones posteriores.
(Taylor, 1892; 256-257). Una de ellas es de significativa importancia para este trabajo. Se trata de la idea (sugerida por Hadamard) de recubrir un conjunto de $V$ incluida la misma clase, mediante una familia de conjuntos o vecindades. En la terminología actual, decimos que Fréchet asume que una familia $\mathcal{M}$ de conjuntos $M$ es una cubierta de un conjunto $E$, cuando cada elemento de $E$ es interior a algún conjunto $M$ de $\mathcal{M}$.

En analogía con los conjuntos de la recta, Fréchet demuestra que para un $\varepsilon>0$, toda clase $V$ separable se puede considerar como la unión de una familia numerable $\mathrm{K}(\varepsilon)$ de conjuntos $k(\varepsilon)$ con la propiedad de que, siendo $A, B$, dos elementos del mismo $k(\varepsilon)$, la vecindad $(A, B)$ es menor que $\varepsilon$. Taylor sugiere designar esta propiedad diciendo que los conjuntos $k(\varepsilon)$ son " $\varepsilon$-pequeños", con lo cual se quiere destacar el sentido moderno del siguiente teorema de Fréchet: Un conjunto $E$ cerrado de una clase $V$ separable y completa (o clase $V$ normal según Fréchet) es compacto si y solo si para cada $\varepsilon>0, E$ es la unión de una familia finita $\mathrm{K}(\varepsilon)$ de conjuntos pequeños $k(\varepsilon)$. (Fréchet, 1906; 25). Esta propiedad que le permite a Fréchet caracterizar un conjunto de una clase $V$ normal como compacto, será utilizada a partir de (Hausdorff, 1914) para definir un conjunto de un espacio métrico como totalmente acotado. (Taylor, 1982; 256-257). A partir de Bourbaki se hizo corriente llamar precompacto a un conjunto totalmente acotado. (Pier, 1980; 440).

Por otra parte, como se sabe, uno de los principales logros de la Tesis de Fréchet es la introducción, bajo el nombre de clase $E$, " $E$ " de écart o distancia, el espacio que años más tarde Hausdorff va a llamar "metrischer Raum" (espacio métrico). (Hausdorff, 1914; 211). La clase $E$ está dotada de una operación binaria tal que a todo par de elementos $A, B$ de la clase asocia un número $(A, B) \geq 0$ que verifica las siguientes propiedades (Fréchet, 1906; p. 30):

(i) La distancia $(A, B)$ es nula cuando $A$ y $B$ son idénticos;

(ii) Si $A, B, C$ son tres elementos cualesquiera, siempre se tiene que $(A, B) \leq(A, C)+(C, B)$.

Luego se demuestra que las vecindades pueden definirse por medio de la distancia, es decir que toda clase $E$ es una clase $V$. Recordemos que un problema de mayor interés en las investigaciones topológicas de los años 1920, empezando por los trabajos de Alexandroff y Urysohn en esta materia, fue el estudio de las condiciones suficientes y necesarias para que una clase $V$ sea clase $E$. Es decir, el problema de las metrizaciones de un espacio abstracto.

La introducción del écart resulta de una forma estructural de pensamiento que busca preservar intuiciones euclidianas en los espacios abstractos. Se define esencialmente por ese modo abstracto de composición entre los puntos del espacio que cumple los axiomas anteriores, y que corresponde al procedimiento estructural del mismo tipo de los modos de composición en la teoría de grupos.

Justamente en relación con este asunto de la influencia de concepciones matemáticas en la constitución de objetos y teorías, cabe tener en cuenta que Fréchet reconoce la 
proximidad entre las concepciones e ideales que sustentaron sus investigaciones en Topología general y Análisis general con la filosofía de Leibniz, en particular con su idea de análisis de los principios. La Noticia sobre sus trabajos científicos lleva el siguiente epígrafe de Leibniz (Fréchet, 1933):

"Quienes prefieren avanzar en los detalles de las ciencias desprecian las investigaciones abstractas y generales. Quienes profundizan en los principios entran raramente en las particularidades. En cuanto a mí, le doy igual importancia a lo uno y lo otro porque he encontrado que el análisis de los principios facilita el avance de las invenciones particulares."

Al inicio de la Noticia manifiesta que se "siente confundido de admiración y de humildad frente a la profundidad de Leibniz y la universalidad de su genio". Considera que, guardadas todas las proporciones, esta cita se aplica a su propio état d'esprit y a la naturaleza abstracta y concreta de sus investigaciones. A lo largo de treinta años, sus trabajos abordaron cuestiones "abstractas y generales" de la Topología, el Análisis funcional y el Análisis general, como también temas más particulares del análisis clásico, la geometría, las probabilidades y la estadística y, últimamente, se había orientado al estudio de las "aplicaciones científicas e industriales de las matemáticas”.

Una sección de la Noticia con sus trabajos "divulgativos y pedagógicos", se refiere a elaboraciones epistemológicas y filosóficas de Fréchet. En (Arboleda y Recalde, 2003) se han examinado y comparado estas ideas con las expuestas en trabajos de años posteriores. Entre ellas se destaca su crítica a la posición de Poincaré sobre intuición y convencionalismo, su elaboración sobre el conocimiento matemático como síntesis inductiva, y sus concepciones sobre axiomatización y "desaxiomatización” en los espacios abstractos.

Retornemos al epígrafe para comentar que aparece literalmente en (Halbwachs, 1906-1928-1950). Se trata de una monografía muy bien documentada en las obras y manuscritos de Leibniz que, sin haber tenido la significación histórica de los trabajos en lógica de Couturat, Russell, Rougier o Nicod, en todo caso pudo permitirle a algunos intelectuales y matemáticos de mentalidad filosófica como Fréchet, familiarizarse con los aspectos fundamentales y sutiles del programa de Leibniz. Maurice Halbwachs (18771945) fue un sociólogo de la escuela de Durkheim que se haría célebre por su teoría sobre la memoria colectiva. Fue colega de Fréchet en el proyecto estratégico de instaurar la enseñanza francesa en la Universidad de Estrasburgo a finales de la primera guerra mundial. Juntos ofrecieron uno de los primeros cursos sobre aplicaciones de la estadística a los fenómenos sociales que se publicaría luego en un pequeño libro: (Fréchet y Halbwachs, 1924).

Su cercanía académica con Halbwachs en Estrasburgo y la lectura de su Leibniz, aportan nuevos elementos para constatar el fondo de la devoción de Fréchet por las ideas de este autor, y ayudan a entender la manera privilegiada en que se posiciona el matemático con respecto a su filosofía desde su propia práctica. Un primer indicio se encuentra en la noción leibniziana de "espacio abstracto" que Fréchet ha podido leer en (Halbwachs, 1928; 93-94). Decir que determinados objetos ocupan ciertas situaciones, equivale a decir que, al existir al mismo tiempo, mantienen entre ellos relaciones más o menos simples. El espacio no es más que el orden de coexistencias posibles, así como la duración no es otra cosa que el orden de situaciones posibles. "Espacio, duración, números, son puros ideales, entia mentalia". La existencia del espacio no es absoluta para Leibniz como lo fuera para Newton; es relativa a la manera como se consideren las relaciones de proximidad entre sus puntos. Esta es una idea central en la caracterización de los espacios abstractos por su estructura, y en la aceptación de distintas estructuras en un mismo conjunto abstracto. (De Risi, 2015), (Tarrés Freixenet, 1994; 197).

Otra expresión de la cita que resulta clave para entender la vocación leibniziana de Fréchet, es el "análisis de los principios". En el capítulo "La Lógica", Halbwachs explica en qué consiste el "arte racional" de Leibniz aplicado a las matemáticas. "Toda demostración o resolución solo es válida si se puede establecer un posible retorno del punto de llegada al punto de partida, por medio de una sucesión de proposiciones idénticas". Fréchet ha podido reconocer aquí el criterio de demostrar una proposición "analizando el sujeto", es decir reduciendo la proposición a una identidad mediante la regla de sustitución de equivalentes.

Tanto más llamativas para Fréchet, lector del Leibniz de Halbwachs, pudieron haber sido las siguientes dos observaciones: 1) Este método es un instrumento efectivo de descubrimiento en matemáticas siempre que la descomposición se lleve hasta su límite y se encuentren en las nociones o proposiciones complejas, las nociones o proposiciones simples. 2) El buen éxito del arte racional depende del descubrimiento de una característica universal, entendida más que como un medio de comunicación entre los hombres de países distintos, "como un alfabeto de nuestras ideas", el "método lógico por excelencia", el sistema de cifras y símbolos que nos permite razonar correctamente y, al mismo tiempo, ganar en economía de pensamiento. (Halbwachs, 1928; 53-54).

Basten estas dos problemáticas filosóficas del Leibniz para ilustrar su proximidad con las concepciones que orientaron el programa de investigación de Fréchet. Este se refiere a ellas en su Noticia (Fréchet, 1934) y en su obra más conocida sobre los Espacios abstractos (Fréchet, 1928), como haciendo parte del sustrato de su programa de unificación de los enunciados clásicos de la Teoría de funciones y del Análisis funcional en el Análisis general. Esta proximidad entre matemáticas y filosofía leibniziana también se puede reconocer en otras esferas de su vida intelectual. Recordemos que durante largos años Fréchet fue promotor y organizador de la Unión Universal de Esperanto, lengua en la cual él mismo publicó interesantes resultados matemáticos a costa incluso de ver por ello reducido su impacto científico. Es posible suponer que Fréchet lo hacía, además de otros intereses humanistas, porque quería convencer a sus colegas 
de que era deseable escoger una lengua "ordinaria" muy general -y acaso más apropiada que otras- para comunicar enunciados matemáticos con carácter universal.

\section{Hausdorff y la formalización de la topología de vecindades}

En sus Elementos de historia de las matemáticas (Bourbaki, 1974; p. 180), Bourbaki reconoce que:

"Con Hausdorff comienza la topología general como se la entiende actualmente. Retomando la noción de vecindad, supo escoger entre los axiomas de Hilbert para las vecindades del plano aquellos que podían dar a su teoría toda la precisión y al mismo tiempo toda la generalidad deseable. El capítulo en el que desarrolla las consecuencias continúa siendo el modelo de teoría axiomática, abstracta pero bien concebida para adaptarse a las aplicaciones. Este fue el punto de partida natural de las posteriores investigaciones sobre la topología general."

En efecto, la axiomática de las vecindades de Hausdorff para la topología de un espacio abstracto se introduce en el capítulo VII sobre "Conjuntos de puntos en espacios generales" de los Fundamentos de la teoría de conjuntos (Hausdorff, 1914). (La selección y traducción de los apartes de Grundzüge der Mengenlehre que se comentan en seguida son tomados de (Dugac, 2003); como se sabe estos apartes difieren en su presentación con respecto a otras ediciones y traducciones del original). Al comienzo del primer parágrafo sobre "Vecindades", Hausdorff aclara así uno de los rasgos característicos de la formalización de las estructuras topológicas, el hecho de fundamentarse en lenguaje conjuntista (Hausdorff, 1914; p. 209):

"La teoría de conjuntos ha celebrado su más bello triunfo en la aplicación a los conjuntos de puntos de los espacios, en la clarificación y la consolidación de los fundamentos geométricos."

Antes de presentar la forma general del sistema de axiomas sobre las vecindades para un espacio abstracto, Hausdorff lo interpreta para un espacio métrico siguiendo la idea originaria de Hilbert que, como veremos, se encuentra en (Hilbert, 1902). Hausdorff procede como si la ilustración del objeto le permitiera justificar la pertinencia de su escogencia (Hausdorff, 1914; p. 211):

"Pero para generar inmediatamente una imagen concreta, comencemos por vecindades especiales que se definen a través de la distancia. Entendemos por espacio métrico un conjunto $E$ en el cual se hace corresponder a dos elementos (puntos) $x$ e $y$ cualesquiera, un número real no negativo, su distancia $\overline{x y} \geq 0$ y además exigimos seguidamente la validez de los axiomas siguientes sobre vecindades:

(a) (Axioma de simetría). Siempre se tiene que $\overline{y x}=\overline{x y}$.

$(\beta)$ (Axioma de coincidencia). Siempre se tiene que $\overline{x y}=$ 0 si y solamente si $x=y$.

$(\gamma)$ (Axioma de desigualdad triangular). Siempre se tiene que $\overline{x y}+\overline{y z} \geq \overline{x z}$.'

Define enseguida una vecindad $U_{x}$ de un punto $x$ en un espacio métrico $E$, como el conjunto de puntos $y$ cuya distancia a $x$ es más pequeña que un número positivo dado. Después de precisar que de acuerdo con la costumbre de sus antecesores emplea la designación de espacio abstracto para indicar que sus elementos son de naturaleza cualquiera, introduce el siguiente sistema de axiomas (Hausdorff, 1914; p. 213):
"Por espacio topológico entendemos un conjunto E, cuyos elementos (puntos) $x$ pertenecen a subconjuntos $U_{x}$ que llamamos vecindades de $x$, y que verifica los siguientes axiomas de vecindades:

(A) Para todo $x \in E$, existe una vecindad $U_{x}$ y $x \in U_{x}$.

(B) Si $U_{x}$ y $V_{x}$ son vecindades de $x$, existe otra vecindad de $x, W_{x}$ tal que $W_{x} \subseteq U_{x} \mathrm{y} W_{x} \subseteq V_{x}$.

(C) Si $y \in U_{x}$, existe un $U_{y}$ tal que $U_{y} \subseteq U_{x}$.

(D) Si $x \neq y$, existen $U_{x}$ y $U_{y}$ tales que $U_{x} \cap U_{y}=\varnothing$."

El axioma (D) caracteriza la condición de separación de los llamados espacios de Hausdorff o espacios $T_{2}$. El estudio de otras modalidades de axiomas de separación es un capítulo interesante en la historia de los espacios topológicos en sus primeros años. Fréchet propuso, en particular, el axioma que caracteriza el espacio topológico $T_{1}$ de Fréchet, en el cual cada par de puntos distintos poseen respectivamente una vecindad que no contiene al otro. Fréchet los denominó "espacios accesibles".

Recordemos que previamente a su obra de 1914, Hausdorff trató las propiedades fundamentales del sistema de vecindades en sus conferencias de Bonn de 1912. Los manuscritos de estas conferencias hacen parte del fondo científico de Hausdorff conservado en la Universidad de Bonn. La transcripción de los apartes de las lecciones correspondientes al sistema de vecindades ha sido publicada, en particular, en (Taylor, 1985) y (Epple, et al., 2002). En ellas se observa que antes de dar una presentación formal del sistema y permaneciendo fiel al enfoque intuitivo que caracterizó sus primeros trabajos matemáticos, Hausdorff prefirió interpretar las vecindades de manera geométrica como el interior de esferas con centro en puntos de un espacio euclidiano $E$ de dimensión $n$. En sus lecciones de 1912 el sistema de axiomas es equivalente al de los Fundamentos de la teoría de conjuntos (1914), salvo el axioma (B) que se enuncia entonces de la siguiente manera: "Dadas dos vecindades de un punto $U_{x}$ y $U_{x}^{\prime}, U_{x} \subseteq U_{x}^{\prime}$ o bien $U_{x} \supseteq$ $U_{x}^{\prime}$ ". Esta cuestión y otras conexas con los fundamentos de la topología de vecindades, han sido tratadas además de (Taylor, 1985) y (Eppel, et al., 2002), por (Sholtz, 1996), (Koetsier y van Mill, 1999) y (James, 1999).

Sabemos que la idea de "topologizar" el espacio euclidiano mediante un adecuado sistema de vecindades se debe a Hilbert, quien lo introduce en sus investigaciones sobre el problema de los fundamentos de la geometría y la continuidad del espacio. Esta idea aparecerá bajo el mismo título, Sobre los fundamentos de la geometría, primero en la memoria (Hilbert, 1902) y luego en la nota (Hilbert, 1903). La última será incluida como apéndice en la traducción de Townsend, (Hilbert, 1902a). Menos conocido es que en este trabajo, Hilbert apela a una argumentación original de la topología conjuntista cantoriana en dos dimensiones, para sustentar su convicción de que la noción de movimiento no era necesaria para fundamentar el sistema de axiomas del espacio euclidiano y que, por consiguiente, su enfoque de la geometría era más general que la teoría de grupos de transformaciones de Helmholtz, Lie y Poincaré. Esta cuestión ha sido analizada en dos contextos distintos en (Majer, 2006) y (Rodríguez, 2015). 
Según la cita de Bourbaki antes mencionada, Hausdorff se inspiró en Hilbert para establecer su sistema de axiomas para las vecindades. Recordemos que (Hilbert, 1902) comienza por considerar el plano como un espacio abstracto constituido por puntos, y pasa a asociarle a cada punto una familia de vecindades o subconjuntos del plano, ello con el interés de caracterizar la continuidad como una propiedad local del espacio. El sistema de vecindades difiere en ambos trabajos de Hilbert. En (Rodríguez, 2015) se analizan estas diferencias desde el punto de vista del problema de la continuidad del espacio que más interesaba a Hilbert y en relación con las propias investigaciones de Riesz a este respecto. En (Hilbert, 1902) el sistema de vecindades está determinado por los siguientes seis axiomas (de acuerdo con la presentación de (Taylor, 1985) que permite evidenciar mejor su relación con el sistema de Hausdorff):

(1) Un punto pertenece a cada una de sus vecindades.

(2) $\mathrm{Si} B$ es un punto en una vecindad $U$ del punto $A$, entonces $U$ es también una vecindad de $B$.

(3) Si $U$ y $V$ son vecindades de $A$, existe otra vecindad de $A$ que está contenida tanto en $U$ como en $V$.

(4) Si $A$ y $B$ son dos puntos cualesquiera, existe una vecindad de $A$ que contiene a $B$.

(5) Para cada vecindad existe al menos una aplicación uno a uno de sus puntos en puntos $(x, y)$ de alguna región de Jordan (el interior de una curva cerrada simple) en el plano numérico.

(6) Dado un punto $A$, una vecindad $U$ de $A$, y una región de Jordan $G$ imagen de $U$, entonces toda región $H$ de Jordan contenida en $G$ y que contiene la imagen de $A$ es también imagen de alguna vecindad de $A$. Si una vecindad de $A$ tiene como imágenes dos regiones de Jordan diferentes, la correspondencia uno a uno inducida es bicontinua.

Hermann Weyl, uno de los primeros en reconocer la importancia del nuevo enfoque tanto para la investigación en topología conjuntista como en su aplicación a la formalización del concepto de variedad, dejó al respecto el siguiente testimonio (Weyl, 1944):

"(...) Hilbert define una variedad de dos dimensiones por medio de vecindades, y requiere que se designe una clase "admisible" de aplicaciones uno-a-uno de una vecindad sobre dominios de Jordan en un plano- $x, y$, tales que cualesquiera dos de ellas estén conectadas por transformaciones continuas. Cuando hice un curso sobre superficies de Riemann en Göttingen en 1912, consulté el trabajo de Hilbert y noté que las mismas vecindades podían usarse para caracterizar tal clase. La definición resultante obtuvo su toque final de F. Hausdorff; los axiomas de Hausdorff se han convertido en un paradigma en topología."

Observemos que en (Hausdorff, 1914, 315) se define un espacio métrico completo como aquel espacio métrico en el cual toda sucesión de Cauchy ("sucesión fundamental") converge. Hausdorff recuerda que Fréchet ha generalizado antes esta propiedad para una "clase". Efectivamente en (Fréchet, 1906; 23) aparece la siguiente definición: decimos que una "clase $V$ admite una generalización del teorema de Cauchy si y solo si toda sucesión de elementos de la clase, que satisface las condiciones de Cauchy, tiene un elemento límite". Hausdorff demuestra que todo espacio métrico $E$ se puede prolongar en un espacio métrico completo $\mathbf{E}$, en donde E resulta ser el conjunto de clases de equivalencia de las sucesiones de Cauchy de $E$ con respecto a la convergencia de sus distancias a cero. En su demostración Hausdorff dice emplear un método análogo al utilizado por $\mathrm{G}$. Cantor y $\mathrm{Ch}$. Méray para construir los números reales. El procedimiento se estudia en (Dugac, 1984; 19-20).

Hausdorff aclara en 1914 que la topología del espacio puede fundamentarse indistintamente en las nociones de distancia, convergencia secuencial o vecindades, pero escoge axiomatizar las vecindades porque la noción de vecindad es más general que la de distancia. Es decir, permite deducir de ella una teoría de conjuntos de puntos válida no solo para la recta y el plano, sino para las superficies de Riemann, los espacios de dimensión finita e infinita y los espacios de curvas y superficies.

En el contexto de las investigaciones sobre estas cuestiones alrededor de los años 1930, el espacio de Hausdorff se revela como "un concepto unificador del pensamiento geométrico" (Alexandroff, 1961; p. 9). En el prefacio de la obra Elementary Concepts of Topology, cuya versión alemana fue publicada en 1932, Hilbert se refiere así a la importancia del formalismo de Hausdorff para la topología de vecindades comparado con la diversidad de investigaciones previas (Alexandroff, 1961):

"Pocas ramas de la geometría se han desarrollado tan rápida y exitosamente en tiempos recientes como la topología, y raramente una rama de una teoría que inicialmente era poco prometedora como la topología, ha adquirido una importancia tan fundamental en un amplio rango de campos completamente diferentes. Actualmente se utilizan los métodos de la topología y se estudian problemas topológicos en casi todas las ramas del análisis y de sus más amplias aplicaciones. Este dominio amplio de aplicaciones requiere naturalmente que la estructura conceptual sea de tal precisión que pueda reconocerse el núcleo común de problemas que en primera instancia parecen distintos."

La precisión de la estructura conceptual era justo lo que buscaba Hausdorff cuando, entre varias presentaciones generales de la teoría, escogía aquella con la axiomática más sencilla y menos redundante posible. En alusión evi-dente a las clases $L$ de Fréchet, afirma que las vecindades eran preferibles "porque permiten desembarazarse de las dificultades de lo numerable que conlleva el uso de la convergencia secuencial" (Hausdorff, 1914; p. 211). Su punto de vista es que la generalidad no debe traducirse en grandes complicaciones $\mathrm{y}$, al menos en lo relacionado con las características principales de la teoría, debe acompañarse de la simplicidad de la presentación.

Este estilo de presentación formal nos protege contra los errores de razonamiento derivados de una intuición defectuosa (Hausdorff, 1914; p. 211):

"Finalmente, con este procedimiento lógico-deductivo nos protegemos de los errores a los que nos puede conducir la intuición. Esta pretendida fuente de conocimiento - cuyo valor heurístico obviamente no se cuestiona- se nos revela a menudo tan insuficiente y 
poco confiable en las partes más sutiles de la topología de conjuntos de puntos, que solo podemos confiar en ella después de un examen cuidadoso."

Este "examen cuidadoso" equivale al "análisis lógico de la intuición espacial" al que se refiere Hilbert en la introducción de los Fundamentos de la Geometría, una obra que, por supuesto, Hausdorff comprendió a fondo desde el comienzo (Punkert, 2008; p. 44-45) (Epple, 2006; p. 279280). La garantía de la precisión conceptual de la estructura topológica es pues, el análisis lógico de la intuición. Es lo que Hilbert reafirma expresamente en la continuación de la cita anterior en el prefacio a los Elementary Concepts of Topology (Alexandroff, 1961):

"No es de extrañar que el análisis de los conceptos geométricos fundamentales les sustraiga en gran medida su inmediata intuición, tanto más, cuando en la aplicación a otros campos, como en la geometría de nuestro espacio circundante, se hace necesaria una extensión a dimensiones arbitrarias."

Fue a finales de la Primera Guerra, cuando se normaliza la actividad matemática y los Fundamentos de la Teoría de Conjuntos pudieron circular ampliamente, que empezó a manifestarse la preferencia por los espacios de Hausdorff. Las lecturas de la obra empezaron a dar sus frutos en numerosas publicaciones de topología conjuntista. El caso más notable fue el grupo de matemáticos que empezaban a liderar las escuelas de Moscú y Varsovia, respectivamente Alexandroff, Urysohn y Tychonoff, y Sierpinski y Kuratowski.

Entre estas contribuciones cabe señalar la memoria (Alexandroff y Urysohn, 1929), que en esencia recogía los resultados de las investigaciones adelantadas por sus autores desde 1922. El propósito de la memoria era remediar la dispersión que prevalecía en la época entre diferentes tratamientos de la noción de compacto en los espacios abstractos. Se trataba entonces de adoptar un lenguaje unificado para sistematizar tales resultados y, al mismo tiempo, seleccionar el espacio topológico más adecuado para los requerimientos de la teoría. En su correspondencia de los años 1920 con Fréchet ambos autores explican el proceso que siguieron para construir una teoría unificada y simple de la compacidad tomando como base el espacio de Hausdorff (al que ellos designan como el "espacio topológico"), en lugar del espacio accesible de Fréchet (Arboleda, 1980a) (Arboleda, 1984).

Concretamente en la carta del 28 de enero de 1924 Alexandroff recuerda que años antes habían introducido la noción de "bicompacto" para reemplazar la compacidad de Fréchet y hacerla más conforme con la definición de la compacidad "ordinaria" (Arboleda, 1979). Se refieren a la definición que utilizaba el teorema de Borel-Lebesgue cuya utilización se hizo corriente a través de los Fundamentos de la Teoría de Conjuntos. Si se reemplaza el término original de "bicompacto" por "compacto" a secas, se evidencia que la definición de la Memoria de 1929 se expresaba en la forma habitual: Un conjunto $E$ es compacto si, dada una familia cualquiera $S$ de conjuntos abiertos que cubre a $E$, un subconjunto finito de $S$ también cubre a $E$.
Anotemos de paso que el anterior cambio de denominación se debe a Bourbaki, quien lo introdujo formalmente en su "Topología general" (Bourbaki, 1971), aunque ya aparece en las versiones anteriores de esta publicación y en otras de miembros del grupo sobre aplicaciones de los teoremas principales de la Memoria de la compacidad a las nuevas nociones de filtros y ultrafiltros (Cartan, 1937a, 1937b) y espacios uniformes (Weil, 1939). La conexión de estos trabajos con la crítica de Bourbaki a lo numerable en topología se estudia en (Arboleda, 1982) y (Anacona, 2017).

La presentación de la compacidad a través de una de las propiedades que hacia los años 1920 mejor expresaba su carácter esencial, el teorema de Borel-Lebesgue, inmediatamente se reveló fecunda y permitió la interrelación con otros conceptos y teorías. En (Arhangel'skii y Dranishnikov, 1997), Arhangel'skii, uno de los alumnos más destacados de Alexandroff, cree encontrar en este tratamiento de la compacidad la manifestación de un rasgo característico de la práctica matemática de su maestro: no proceder jamás con estrechez de miras en la consideración de nociones y construcciones fundamentales; por el contrario, utilizarlas como base para profundizar en la indagación matemática y promover el desarrollo integral de la teoría objeto de estudio. De ahí el papel fundamental que desempeñan los trabajos pioneros de Alexandroff sobre la compacidad y los fundamentos de la topología general, en principios básicos de las matemáticas. Entre los cuales, Arhangel'skii y Dranishnikov prefieren mencionar el teorema de Hahn-Banach, el teorema de Krein-Milman, el teorema de Gel'fand-Kolmogorov, y el teorema de Stone-Weierstrass.

\section{Conclusiones}

La axiomatización de la topología de espacios abstractos es un paso fundamental en el desarrollo de las estructuras matemáticas del siglo XX. Las vecindades constituyen un medio conveniente para seleccionar y organizar las estructuras de la topología general, y garantizar el estudio fecundo de los problemas más importantes de la teoría de funciones.

Fréchet, Hilbert, Riesz, Moore y otros matemáticos hicieron aportes significativos a la caracterización de la topología del espacio abstracto a través de las vecindades. Pero la presentación axiomática que hoy utilizamos fue formulada inicialmente por Hausdorff en sus conferencias de Bonn de 1912 y luego en sus Fundamentos de la teoría de conjuntos (Hausdorff, 1914).

Las propuestas de ambos autores para la fundamentación de la topología general se inspiraron en concepciones filosóficas que fueron determinantes en sus respectivas prácticas matemáticas constitutivas de la teoría. En el caso de Fréchet las estructuras topológicas se introducen y estudian como parte del programa del Análisis general. Este enfoque se reclama de la "analiticidad" leibniziana, y está orientado por un cierto ideal de "categoricidad de los espacios abstractos". En términos de las primeras investigaciones de comienzos del siglo XIX de Hadamard y Fréchet en Análisis 
funcional, este enfoque analítico consistió en extender los métodos conjuntistas cantorianos a una gran variedad de nuevos espacios abstractos.

Cincuenta años después con el advenimiento de la teoría de categorías, la polaridad de lo analítico conjuntista será sustituida por su contraparte sintética: "la teoría de categorías deja de desmenuzar los objetos por dentro y de analizarlos mediante sus elementos, y pasa a elaborar aproximaciones sintéticas en las cuales los objetos son estudiados por su comportamiento exterior en correlación con su medio ambiente". (Zalamea, 2009).

Por su parte, el estilo de Hausdorff se inspira en sus trabajos filosóficos tempranos. Según Hausdorff, entre varias presentaciones generales de la topología del espacio, se escoge aquélla con la axiomática más sencilla y menos redundante posible. Simplicidad, unidad y economía de pensamiento configuran el estilo que hace de los Fundamentos de la Teoría de Conjuntos una obra pionera en su campo y que contribuyó al desarrollo del enfoque estructural de las matemáticas modernas.

\section{Conflicto de intereses}

Ninguno

\section{Referencias}

Alexandroff, P. (1961). Elementary Concepts of Topology. Dover: New York. (Traducción de Einfachste Grundbegriffe der Topologie. Springer, 1932).

Alexandroff, P. S, Fedorchuk, V. V. (1978). The Main Aspects in the Development of Set-Theoretical Topology. Russian Math. Surveys. 33: 1-53.

Alexandroff, P. S., Urysohn, P. S. (1929). Mémoire sur les espaces topologiques compacts. Ver. Kon. Akad. Wetensch. Amsterdam. 14: 1-96.

Anacona, M. P. (2017). La teoría de conjuntos y los números reales en el estructuralismo de Bourbaki. Tesis Doctoral. Cádiz: Universidad de Cádiz.

Arboleda, L. C. (1979). Les debuts de l'École topologique soviétique: notes sur les lettres de Paul S. Alexandrov et Paul S. Urysohn à Maurice Fréchet. Archive for History of Exact Sciencies. 32: 73-89.

Arboleda, L. C. (1980a). Contribution à l'étude des premières recherches topologiques d'après l'œuvre et la correspondance de Maurice Fréchet. Tesis de Doctorado. École d'Hautes Études en Sciences Sociales. Paris.

Arboleda, L. C. (1980b). Las primeras investigaciones sobre los espacios topológicos. Sociedad Colombiana de Matemáticas. X Coloquio Colombiano de Matemáticas, Paipa.

Arboleda, L. C. (1982). Consideraciones metodológicas sobre el aporte de M. Fréchet a la topología general. Actualités mathématiques: actes du VIe Congrès du regroupement des mathématiciens d'expression latine. Paris: GauthierVillars; 493- 499.

Arboleda, L. C. (1984). Sobre los fundamentos de la teoría de los espacios compactos. Asclepio, Madrid. 35: 123-157.

Arboleda, L. C. (2007). Modalidades constructivas y objetivación del cuerpo de los reales. Revista Brasileira de Historia da Matemática, Especial nº 1: 215-230.
Arboleda, L. C., Recalde, L. C. (2003). Fréchet and the Logic of the Constitution of Abstract Spaces from Concrete Reality. Synthese. 134: 245-272.

Arboleda, L. C. (2012). Objetos Matemáticos y Prácticas Constitutivas: La Génesis de la Topología de Vecindades. Notae Philosophicae Scientiae Formalis. 1: 32-44.

Arhangel'skii, A. V. y Dranishnikov, A. N. (1997). P. S. Alexandroff and Topology: an introductory note. Topology and its Applications, 80: 1-6.

Aull, C. E., Lowen, R. (eds.) (1997). Handbooks of the History of General Topology. Vol.1. Dordrecht: Springer.

Bourbaki, N. (1971). Topologie Générale. Vol. 1, chapitres 1 à 4. Paris: Hermann.

Bourbaki, N. (1974). Éléments d'histoire des mathématiques. Paris: Hermann.

Cartan, H. (1937a). Théorie des filtres. C. R. Acad. Sci. Paris. 205: 595-598.

Cartan, H. (1937b). Filtres et ultrafiltres. C. R. Acad. Sci. Paris. 206: $777-779$.

De Risi, V. (ed.) (2015). Mathematizing Space. The Objects of Geometry from Antiquity to the Early Modern Age. Heidelberg: Birkhäuser.

Dieudonné, J. (1981). History of functional analysis. Amsterdam: North-Holland.

Dugac, P. (1984). Histoire des espaces complets. Revue d'Histoire des Sciences. 37: 3-28.

Dugac, P. (2003). Histoire de l'Analyse. Autour de la notion de limite et de ses voisinages. Paris: Vuibert.

Epple, M., Herrlich, H., Husek, M., Preuß, G., Purkert, W., Scholz, E. (2002). Zum Begriff des topologischen Raumes. En: Felix Hausdorff: Gesammelte Werke, Bd. II. Berlin: Springer.

Epple, M. (2006): Felix Hausdorff's Considered Empiricism. En J. Ferreirós, J. Gray (Ed) (2006): The Architecture of Modern Mathematics (263-289). New York: Oxford University Press.

Fréchet, M. (1904). Généralisation d'un théorème de Weierstrass. C. R. Acad. Sci. Paris. 139: 848-850.

Fréchet, M. (1906). Sur quelques points du calcul fontionnel. Rendiconti Circolo Mat. Palermo. 22: 1-74.

Fréchet, M. (1928). Les Espaces Abstraits et leur théorie considérée comme introduction à l'Analyse Générale. Paris: GauthierVillars.

Fréchet, M. (1933). Notice sur les travaux scientifiques de Maurice Fréchet. Paris: Hermann.

Fréchet, M. (1941). L'analyse générale et la question des fondements. En: Gonseth, F. (ed). (1941). Les entretiens de Zurich sur les fondements et la méthode des sciences mathématiques. Zurich: Leemann. p. 53-81.

Fréchet, M. et Halbwachs, M. (1924). Les calcul de probabilités à la portée de tous. Paris: Dunod.

Hadamard, J. (1912). Le calcul fonctionnel. Enseignement mathématique. 14: 5-18.

Halbwachs, M. (1906). Leibniz. Collection Les Philosophes. Paris: Delaplane. Segunda edición aumentada: Paris, Mellotée, 1928. Tercera edición: Paris, Mellotté, 1950.

Hausdorff, F. (1914). Grundzüge der Mengenlehre. Leipzig: Veit.

Hilbert, D. (1897). Zum Gedächtnis an Karl Weierstrass. En D. Hilbert (1970). Gesammelte Abhandlungen (vol. 3). Berlin: Springer-Verlag.

Hilbert, D. (1899). Grundlagen der Geometrie. Leipzig: Teubner. 
Hilbert, D. (1902): Über die Grundlagen der Geometrie. Göttinger Nachrichten. 4: 233-241.

Hilbert, D. (1902a). The Foundations of Geometry. Chicago: Open Court.

Hilbert, D. (1903): Über die Grundlagen der Geometrie. Math. Annalen. 56: 381-422.

Hirsch, G. (1978). Topologie. En J. Dieudonné. (Ed). Abrégé d'Histoire des mathématiques. 2: 211-266. Paris: Hermann.

James, I. M. (Ed) (1999). History of Topology. Amsterdam: Elsevier.

Katz, V., Parshall, K. H. (2014). Taming the Unknown: A History of Algebra from Antiquity to the Early Twentieth Century. Princeton: Princeton University Press.

Koestsier, T. Y Van Mill, J. (1999). By their fruits ye shall know them: Some remarks on the interaction of general topology with other areas of mathematics. En I. M. James (1999): 199-239.

Majer, U. (2006). The relation of Logic and Intuition in Kant's Philosophy of Science, particularly Geometry. Western Ontario series in Philosophy of Science. 70: 47-66.

Manheim, J. (1964). The Genesis of point set Topology. Rinehart: Oxford.

Purkert, W. (2008). The Double Life of Felix Hausdorff/Paul Mongré. Math. Intellingencer. 30: 36-50.

Pier, J.-P. (1980). Historique de la notion de compacité. Historia Mathematica. 7: 425-443.
Rodríguez, L. (2015). Frigyes Riesz and the emergence of general topology. The roots of 'topological space' in geometry. Archive for History of Exact Sciences, 69: 55-102.

Scholz, E. (1996). Logische Ordnungen im Chaos: Hausdorffs frühe Beiträge zur Mengenlehre. En E. Brieskorn (Ed) (1966). Felix Hausdorff zum Gedächtnis, Vol. 1, Aspekte seines Werkes (107-134). Wiesbaden: Vieweg.

Séguier, J. A. de (1904). Théorie des groupes finis. Éléments de la théorie des groupes abstraits. Paris: Gauthier-Villars.

Tarrés Freixenet, J. (1994). La topología general desde sus comienzos hasta Hausdorff. Historia de la matemática en el siglo XIX (2 ${ }^{\text {a }}$. Parte). Madrid: Real Academia de Ciencias Exactas, Físicas y Naturales.

Taylor, A. E. (1982). A Study of Maurice Fréchet: I. His Early Work on Point Set Theory and the Theory of Functionals. Archive for History of Exact Sciences. 27: 233-295.

Taylor, A. E. (1985). A Study of Maurice Fréchet: II. Mainly about his Work on General topology, 1909-1928. Archive for History of Exact Sciences. 34: 279-380.

Weil, A. (1937). Sur les espaces à structure uniforme et sur la topologie générale. Publications de l'Institut de mathématiques de l'Université de Strasbourg. Paris: Hermann.

Weyl, H. (1949). David Hilbert and his Mathematical Work. Bulletin of American Mathematical Society. 50: 612-654.

Zalamea, F. (2009). Filosofía sintética de las matemáticas contemporáneas. Bogotá: Universidad Nacional de Colombia. 